\title{
Maternal Mortality Trend in Ethiopia
}

\author{
Ahmed Abdella
}

\begin{abstract}
Background: Maternal mortality ratio is one of the indicators in the MDG that is raising concern in achieving the set target of reducing the rate by two-third by 2015 .

Objectives: To review the maternal mortality trend and the causes of maternal deaths in Ethiopia.

Methods: Review of published and unpublished documents addressing maternal mortality in Ethiopia.

Result: DHS and hospital data indicate decreasing maternal mortality even though the current maternal mortality ratio is still high. The proportion of maternal deaths due to the each of the five major causes varies with time. Generally, the limited information indicates that the proportion of maternal deaths after unsafe abortion is decreasing while deaths after preeclampsia/ eclampsia are increasing. There is no grossly notable change in the proportion of deaths due to ruptured uterus/ obstructed labor, hemorrhage and sepsis. In recent studies, maternal deaths following hepatitis are not reported while deaths due to HIV are appearing. Deaths complicated by malaria are seen in certain parts of the country. The case fatality rates of preeclampsia/eclampsia and ruptured uterus/ obstructed labor are increasing.

Conclusion: Even though the review is constrained by inadequate data and interpretation uncertainty of the findings, it generally indicates the urgent need of improving the quality of maternal health services; scaling up evidence based interventions; and measuring progress. [Ethiop. J. Health Dev. 2010;24 Special Issue 1:115-122]
\end{abstract}

\section{Introduction}

Globally, at least 585, 000 women die each year by complications of pregnancy and child birth (1). More than $70 \%$ of all maternal deaths are due to five major complications: hemorrhage, infection, unsafe abortion, hypertensive disorders of pregnancy, and obstructed labor. The majority of maternal deaths (61\%) occur in the postpartum period, and more than half of these take place within a day of delivery (2).

The poor health and nutrition of women and the lack of care that contributes to their death in pregnancy and child birth also compromise the health and survival of the infants and children they leave behind. It is estimated that nearly two-third of the 8 million infant deaths that occur each year largely from poor maternal health and hygiene, inadequate care, inefficient management of delivery, and lack of essential care of newborn (1).

An estimated $40 \%$ of pregnant women (50 million per year) experience pregnancy-related health problems during or after pregnancy, and childbirth, with 14\% suffering serious or long term complications. As a consequence, 300 million women suffer from pregnancyrelated health problems and disabilities, including anemia, uterine prolapse, fistula, PID, and infertility (2).

There are many factors that affect the outcome of pregnancy from the onset of any obstetric complication. The outcome is most adversely affected by delayed treatment. Delay in treatment is the result of many factors. These delays are described as the three phases of delay (3):

Delay I: Lack of information and adequate knowledge about danger signals during pregnancy and labor; cultural/ traditional practices that restrict women from seeking health care; lack of money
Delay II: Out of reach of health facilities; poor road, communication network, community support mechanisms

Delay III: Inadequate skilled attendants; poorly motivated staff; inadequate equipment and supplies; weak referral system, procedural guides.

The three main delay factors (distance, cost, and quality) are ultimately defined by illness-related behavior. Their salient as obstacles is ultimately defined by illnessrelated factors, such as severity. The gravity of the illness is often the major consideration in the decision to seek care. Broadly also, the use of health resources is shaped by the context in which individuals live: culture/ tradition/laws, distribution of health facilities. Almost $90 \%$ of the maternal deaths occur in Sub-Saharan Africa and Asia, making maternal mortality the health statistic with the largest discrepancy between developed and developing countries. While women in north Europe have a 1 in 4,000 likelihood of dying from pregnancyrelated causes, for those in Africa the chance is 1 in 16 (2).

The tragedy is that these women die not from disease but during the normal, life-enhancing process of procreation. Most of these deaths could be avoided if preventive measures were taken and adequate care was available. Maternal death is an indicator of disparity and inequity between men and women and its extent a sign of women's place in society and their access to social, health, and nutrition services and to economic opportunities (1).

The fifth Millennium Development Goal (MDG) aims at improving maternal health and targets reducing maternal mortality ratio (MMR) by $75 \%$ between 1990 and $2015-$ that is, it seeks to achieve an expected $5.5 \%$ annual decline in MMR from 1990. However, MMR has

Obstetrics and Gynaecology Department, Medical Faculty, Addis Ababa University, P. O. Box: 1752, Addis Ababa-

1110, Ethiopia, E-mail: ahmedamu@yahoo.com 
decreased at the global level at an average of less than $1 \%$ annually between 1990 and 2005. To make the achievement of the fifth MDG a reality, MMR will have to decrease at a much faster rate - especially in subSaharan Africa (including Ethiopia), where the annual decline is staggering behind (4).

For monitoring progress towards achievement of maternal mortality reduction, MMR and the proportion of births attended by skilled health personnel are the indicators. Country estimates of maternal mortality (and their causes) over time are crucial to inform planning of sexual and reproductive health programs and to guide advocacy efforts and research at the national level, particularly within the context of the MDG. These estimates are also needed at the international level, to inform decision-making concerning resource allocation by development partners and donors. However, assessing the extent of progress towards the MDG5 target has been challenging, due to the lack of reliable maternal mortality data - particularly in developing-country settings where maternal mortality is high (4). In this review, available documents were analyzed to assess the trend of maternal mortality and it's causes.

\section{Methods}

Available documents addressing maternal mortality were identified and content review was undertaken. The main sources considered in the document search were national medical journals and documents. The three main health journals (Ethiopian Medical J., Ethiopian J. Health Development and Ethiopian J. Reproductive Health) published since the late 1970 were manually searched. Limited search for student research papers available in the Medical Faculty of Addis Ababa University and internet search (using key words of 'maternal mortality death and Ethiopia') was made to identify relevant and accessible publications. References of available papers were also used to identify other possible publications.

A thorough review of the identified documents was made following an initial assessment of their titles and abstracts or summary. The available data was initially captured using a data extracting format and hand notes. The data was later entered into a 'Microsoft Excel Spreadsheet'. The soft ware was also used to analyze and depict graphs.

The main variables collected include maternal mortality ratio (MMR), causes of maternal deaths, case fatality ratio (CFR), year of study, place of study, type of study (community or hospital), and underlying factors whenever available. When a certain proportion such as MMR or CFR is not reported but the required raw data are provided in a document, the required proportion is generated using the raw data. If a study covers more than one year, the mid-year is used as the 'year of study'.

To have a glimpse on the recent situation, a review of maternal deaths (MD) during two years (2007 to 2009) at Tikur Anbessa Hospital (TAH) and Gandhi Memorial Hospital (GMH) was undertaken. Results from this review were cited as unpublished (reference 13).

\section{Results}

Based on the 2000 and 2005 Ethiopian DHS findings (5), the MMR showed a decreasing trend from 871 to 673 MD/ 100,000 LB (Fig 1) (6). Similarly, the data from various hospitals in the country generally indicated a decreasing MMR trend (Fig 2). On the other hand, the overall MMR trend depicted from difference national (DHS) and community (urban and rural) shows an increasing trend (Fig 1). The MMR estimates from hospital population (ranging from $\approx 500$ to 2500 ) are higher than community based estimates (ranging from $\approx$ 600 to 800$)$.

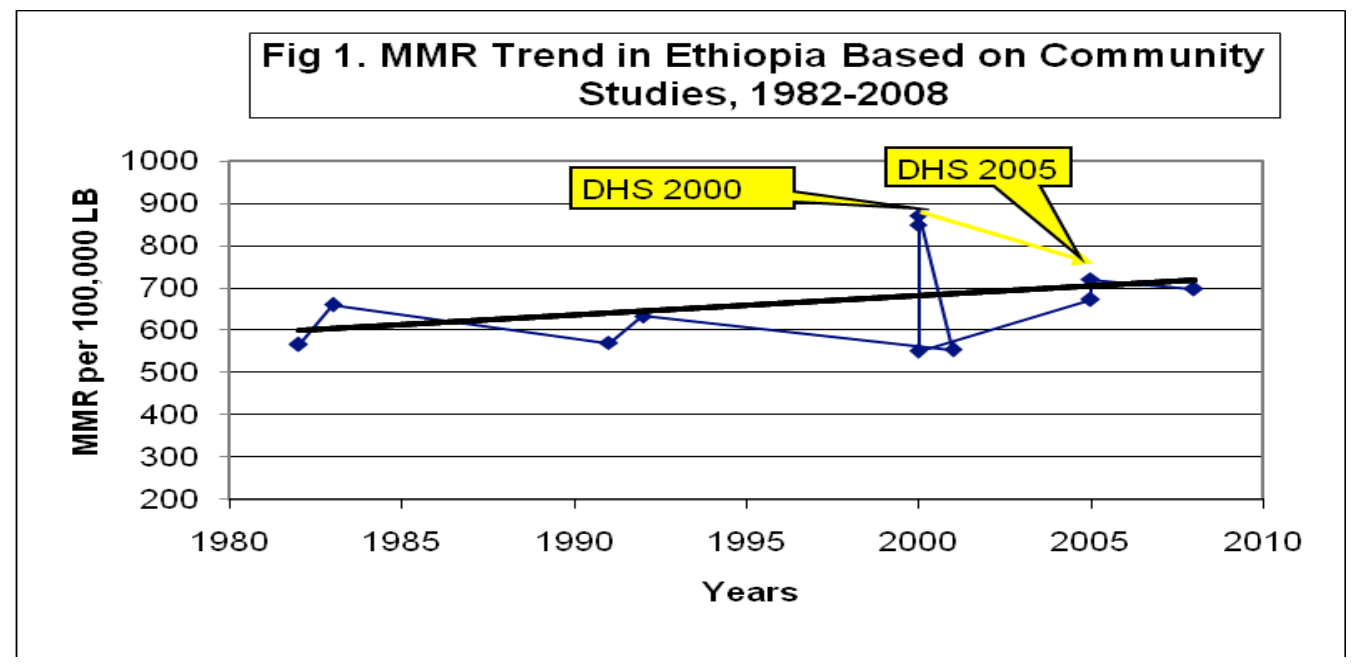

References: 4, 5, 6, 8, 20, 29, 30, 31, 32, 33

Figure 1: MMR Trend in Ethiopia Based on Community Studies, 1982-2008 


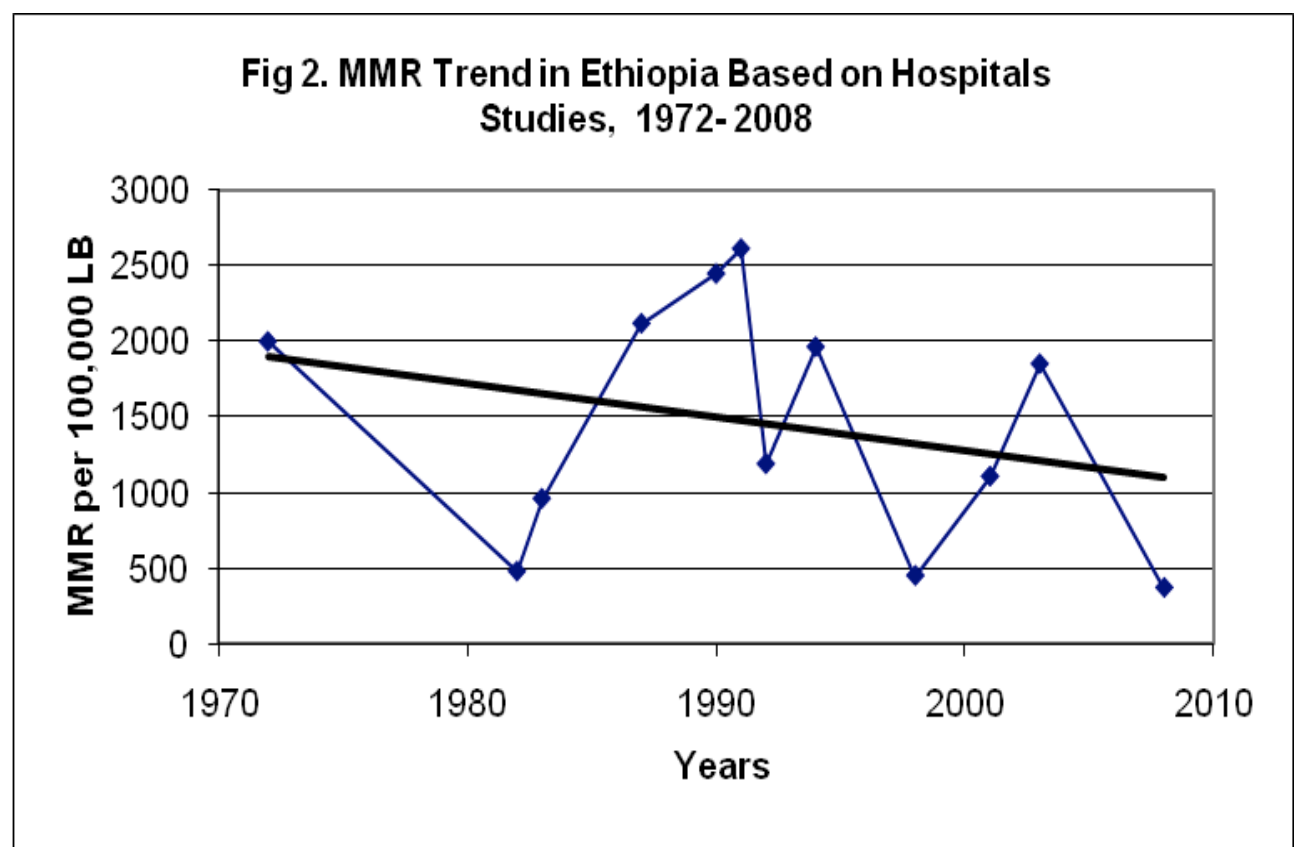

References: $6,7,9,11,12,13,14,21,34,35,36,37$

Figure 2: MMR Trend in Ethiopia Based on Hospitals Studies, 1972-2008

The major causes of maternal deaths in Ethiopia are similar to most developing countries: infection, hemorrhage, obstructed labor, abortion and hypertension in pregnancy. The proportion of MD ascribed to the different causes varies from year to year (Fig 3,5).

Abortion: Maternal death by abortion generally shows a declining trend. Hospital studies before 2003 indicated that the proportions of abortion deaths were the highest among the five major causes of MD; then after, the proportions become one of the lowest (Fig 3). In earlier hospital and community studies, abortion deaths account for $20-50 \%$ of the deaths $(7,8,10,11,12)$. From 2007 to 2008, at TAH and GMH, there were 3 deaths after abortion among $42(7 \%)$ of all maternal deaths (13). In the same two hospitals, from 1981-82, there were 37 deaths due to abortion accounting for $26.6 \%$ of all the maternal deaths (9). In Jimma Hospital, abortion used to account for more than $40 \%$ of all the maternal deaths in the 1980s $(7,12)$; in the 1990s, the proportion of unsafe abortion deaths decreased to $26.8 \%$ (36).

Eclampsia: Unlike deaths of abortion, the proportion of deaths due to eclampsia/ preeclampsia is on the increase (Fig 3,5 ). In the last two years, there were 15 deaths due to eclampsia/ preeclampsia accounting for $35.7 \%$ of the maternal deaths at TAH \& GMH (13). In the same two hospitals, in 1981-1983, there were 9 deaths due to eclampsia which account for $6.5 \%$ of the total MD (9). The prevalence of eclampsia (number of women with eclampsia/ total deliveries) in Addis Ababa hospitals doesn't show a marked increment; it varies from $1.2 \%$ to $7.1 \%$ with most of the studies indicating a prevalence rate of about $3.3 \%(12,14,15,16,17)$.

Previously conducted the proportions of maternal deaths due to uterine rupture/ obstructed labor, and sepsis remain unchanging. Similarly, the proportions of MD following hemorrhage are unchanging, except the recent two reports from Ambo Hospital (34\%) (18) and TAH/ GMH (17.9\%) (13) (Fig 3,5$)$.

Earlier community and hospital studies in Addis Ababa indicate that infectious hepatitis is a common cause of death accounting for $13-15 \%$ of the maternal deaths (8, 11), but none of the recent documents report hepatitis. On the other hand, HIV is appearing in some recent hospital and community studies accounting for $3-4 \%(12,20)$ of all maternal deaths. MD following malaria are reported for $0.05 \%$ (21) to $23.3 \%$ (20) of the deaths in Jimma and Tigray (Fig 5).

Overall the case fatality rates of ruptured uterus/ obstructed labor and prececlampsia/eclampsia indicate an increasing trend while that of abortion remain stable (Fig 4). 


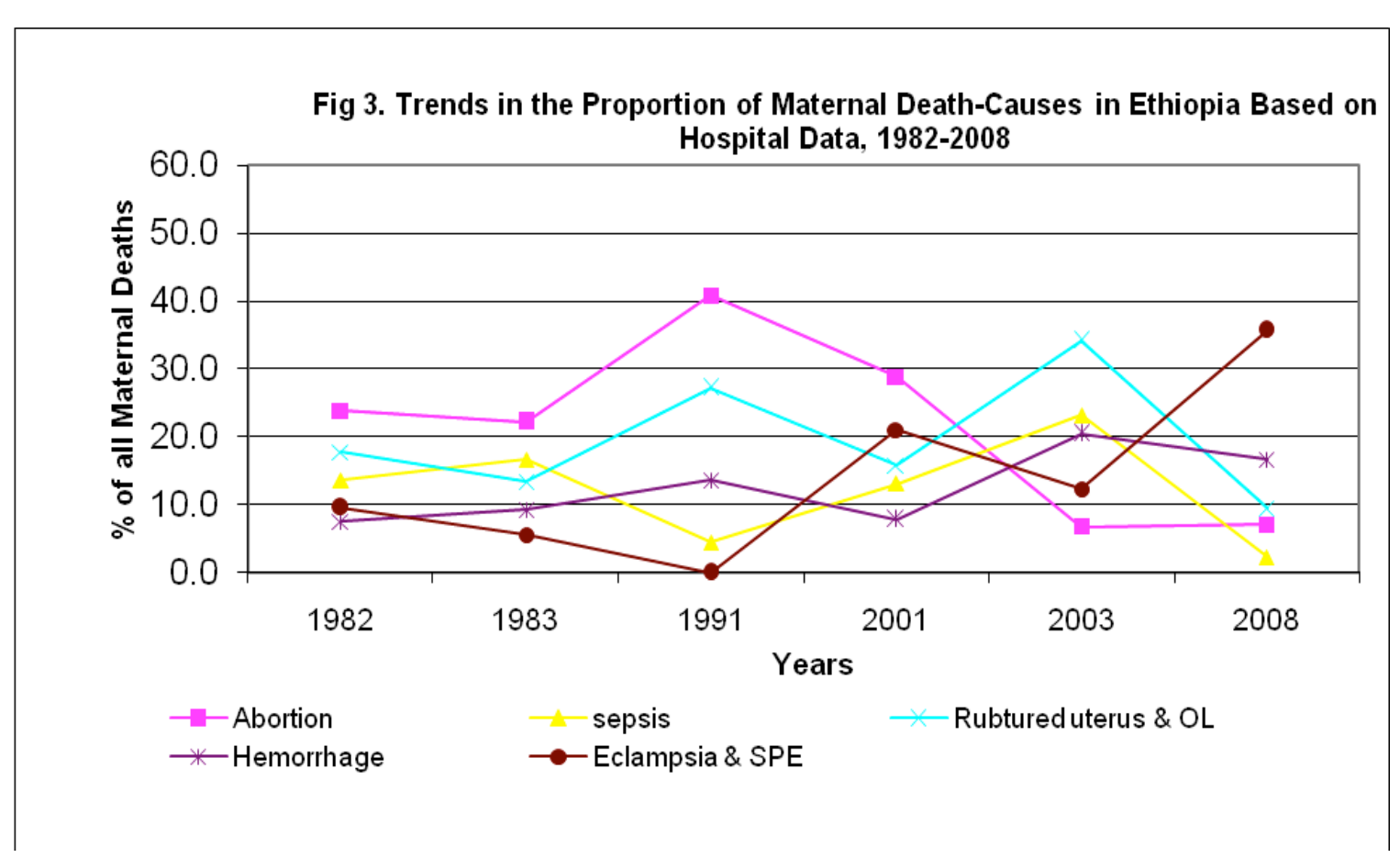

References: 7, 9, 12, 13, 14, 16, 18, 19, 21, 34, 36, 39, 45, 43, 44

Figure 3: Tends in the Proportion of Maternal Death-Causes in Ethiopia Based on Hospital Data, 1982-2008

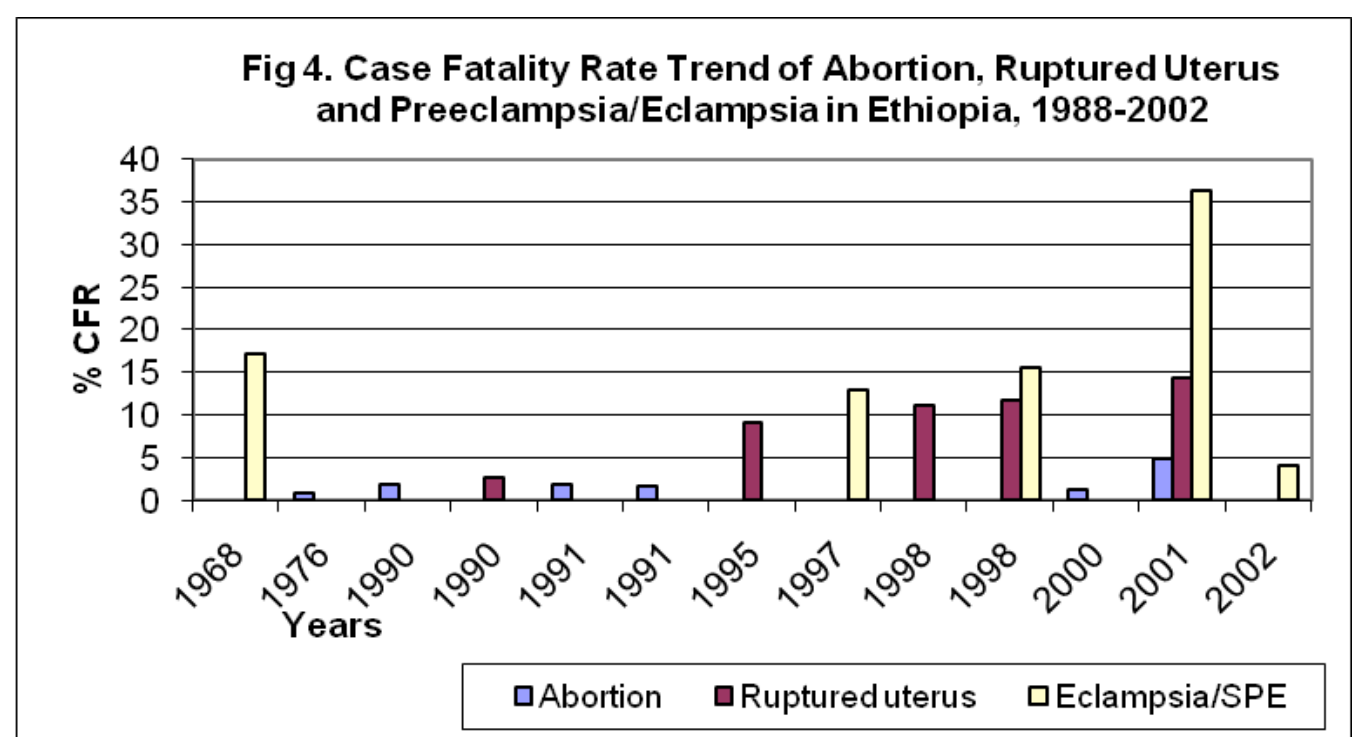

References: 7, 9, 11, 12, 16, 34, 36, 39, 40, 41, 42, 43, 44

Figure 4: Case fatality Rate Trend of Abortion, Ruptured Uterus and Preeclampsia/Eclampsia in Ethiopia. 19882002 


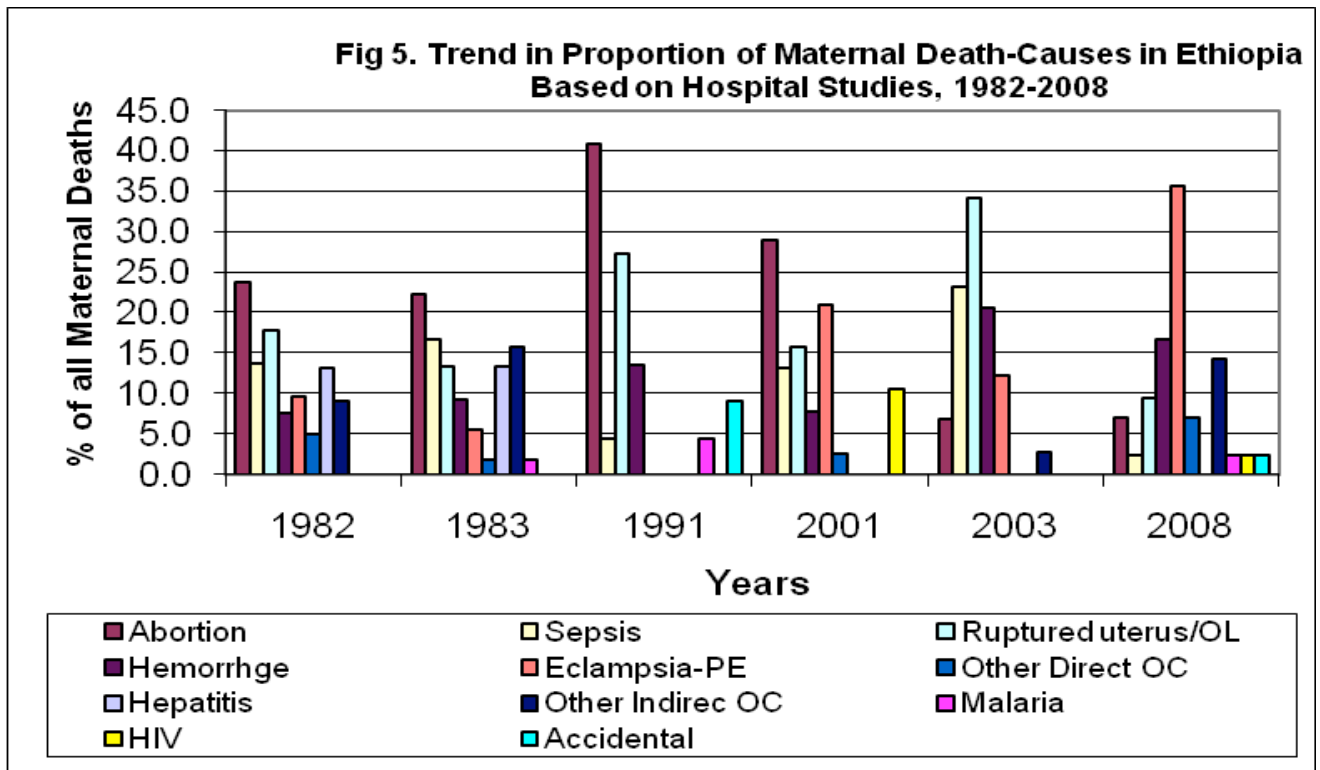

PE; Preeclampsia; OC: obstetric cause of maternal deaths; OL: Obstructed labor

References: 7, 8, 11, 12, 13, 18, 21, 34, 35, 36, 38, 39

Figure 5: Trend in Proportion of Maternal Death-Causes in Ethiopia Based on Hospital Studies, 1982-2008

The analysis of causes of maternal mortality by residence among 39 maternal deaths at TAH/GMH in 2 years indicates that most of the abortion ( 2 of 3 ) and ruptured uterus ( 3 of 4 ) deaths are coming mainly from out of Addis Ababa while most of the maternal deaths due to eclampsia (12 of 13), hemorrhage (5 of 7), infection (all 3 ) and pulmonary embolism (all 3) are from Addis Ababa. Most of the women coming from outside of Addis Ababa (7/10) were in shock on arrival to TAH while non-of the maternal deaths from Addis Ababa were in shock (13).

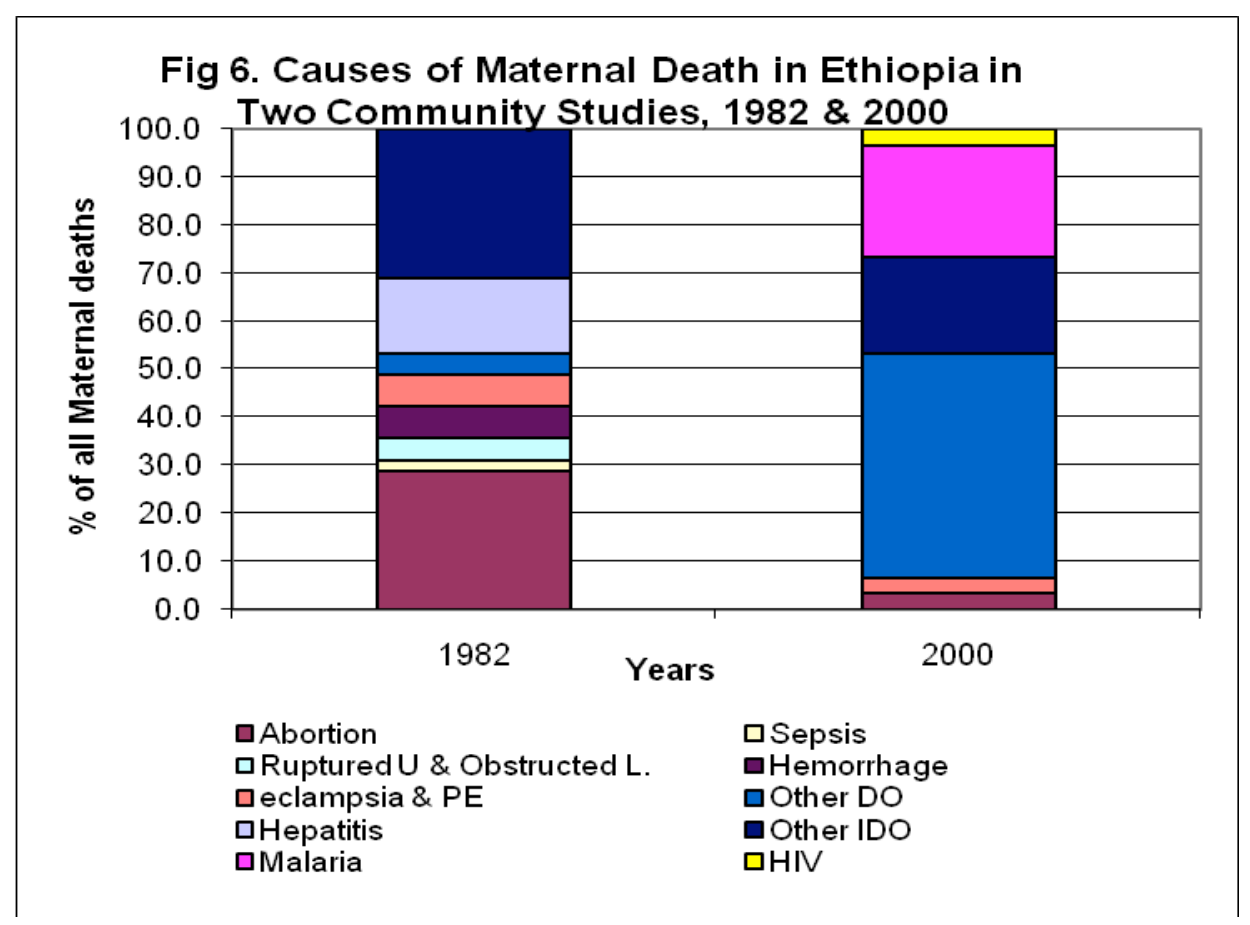

Source: 8,20

Figure 6: Causes of Maternal Death in Ethiopia in two community Studies, 1982 \& 2000 


\section{Discussion}

The maternal mortality trend as implicated by the estimates of DHS and hospital based studies indicate a decreasing trend, unlike the increasing trend depicted by the overall community based studies which includes DHS data sources. Even though the DHS based trend indicates decreasing trend, the current MMR estimate of 673 $\operatorname{MD} / 100,000 \operatorname{LB}(5,6)$ is still a high ratio The high MMR from hospitals than national or community based estimates is mainly due to high proportion of obstetric complication seen among hospital population.

In some of the reviewed papers, total deliveries were used in place of live births (LB) to calculate MMR. Errors in MMR estimations and their interpretations are inherent problems. Generally MMR estimates are subject to high levels of uncertainty and not intended to serve as precise estimates. Caution is required in interpreting small numerical differences in countries as representing real differences in maternal mortality because the estimated uncertainty margins are extremely wide. However, the estimates are indicative of the extent of the maternal mortality problem, and should draw attention to the need for both improved action for maternal mortality reduction and increased efforts for the generation of robust data for estimating maternal mortality levels (4).

To achieve a three-fourth decline in MMR by 2015 as set by MDG, efforts has to be strengthen to address the underlying causes of maternal deaths. Among the major five obstetric causes of maternal deaths, the proportion of maternal deaths due to eclampsia/ severe preeclampsia shows increasing trend while that of abortion is decreasing. No grossly notable reduction in the proportions of MD after ruptured uterus/ obstructed labor and sepsis are indicated.

Currently, almost all women in Ethiopia with eclampsia/ preeclampsia are treated with diazepam while the evidence shows that magnesium sulfate is the best drug for preeclampia/eclampsia treatment. Besides improving the prenatal and delivery care, availing magnesium sulfate as part of emergency obstetric care is of paramount importance.

The decreasing trend in abortion is also support by the high morbidity associated with unsafe abortion in earlier studies. In 1980-1983 and 1989-1992, studies have documented that unsafe abortion accounts for more than $50 \%$ of the admission to the renal unit of TAH $(22,23)$. The relative improvement in access to safe abortion services following the revision of the Ethiopian Penal Code in 2005 (24), and the provision of the 'Technical and Procedural Guideline for Safe Abortion in Ethiopia' in 2006 (25) might have contributed to the declining proportion of unsafe abortion MD.

The proportions of hemorrhage, ruptured uterus/ obstructed labor and sepsis generally remain unchanging
(Fig 3, 5). Maternal death due to postpartum hemorrhage (PPH) evolves fast. It is estimated that a woman dies within two hours of developing postpartum hemorrhage. To avert deaths due to PPH at community level, the use of misoprostol at community level might be considered.

The high proportion of MD due to hepatitis in earlier studies is supported indirectly by the high mortality of pregnant women among medical admission in earlier studies $(26,27.28)$. Maternal deaths due to hepatitis are disappearing in recent studies while deaths due to HIV are appearing. Malaria is also noted in some of the regions as a major cause of maternal death. Malaria might be an important cause of MD in malarious areas.

The increasing trend and no change in the case fatality rates documented for ruptured uterus/ obstructed labor, preeclampsia/eclampsia and abortion indicate the deterioration of (lack of improvement in) the quality of maternal health care provision. The trend in case fatality rate of abortion is generally stable between 0.9 to $1.9 \%$ (except an exceptional recent report from TAH of $4.9 \%$ (12). It has to be noted that most of the studies are done in public facilities where almost all cases seen are abortion with complications and little safe abortion services.

There are a number of data quality issues in the various documents: Besides missing $\mathrm{MD}$, it is common to misclassify MD due to ruptured uterus, obstructed labor, and abortion with hemorrhage and sepsis. The review of $24 \mathrm{MD}$ at TAH reveals that $12(50 \%)$ of them had infection complications such as pneumonia, PID, HIV, TB while 3 of the MD were classified as sepsis (13). Underreporting in deaths associated with HIV is likely as most women's HIV status is unknown. Another source of underestimation of PPH in hospital studies inherent in the fast deterioration of a woman with $\mathrm{PHH}$. Women with postpartum hemorrhage are very unlikely to reach to hospital and appear in hospital statistics if PPH occurs at home delivery.

Even though assessing the different and interrelated delay factors is not the main objectives of this review, the effect of distance on MD in Ethiopia becomes apparent in the process of reviewing the documents on maternal deaths in Ethiopia. In Tigray, a community study found that $80 \%$ of the maternal deaths took place at home. In more than $50 \%$ of the deaths "Poor health seeking behaviors" and lack of transportation were described as the obstacles to access services (20). Women from distant areas arrive at hospitals in morbid clinical condition after a protracted obstetric complication. At Adigrat Hospital where $85 \%$ of MD occurred among rural women, the average duration of labor among women with ruptured uterus was about 60 hours, and about 80 hours among those who died due to rupture (34). Most of the women coming from outside of Addis Ababa (7/10) were in shock on arrival at TAH while non-of the maternal deaths

Ethiop. J. Health Dev. 2010;24 Special Issue 1 
from Addis Ababa were in shock (13). In a study at Jimma Hospital, the case fatality rate of abortion among women from outside Jimma town (3.6\%) was significantly higher (p-value: 00510) than the case fatality rate of women from the town $(1.2 \%)(7)$.

Distance can be a major obstacle to obtain care, and distance can discourage people from even trying to reach care. In general as distance to a facility increases, utilization of its services decreases. The total actual cost of obtaining care is also another important obstacle as it involves the opportunity costs of time, the cost of transportation, cost of drugs and the cost of supplies. Further more the perceived quality of care is another factor affecting the decision to seek care. Poor-quality services deter people from traveling to a facility because experience has taught them that supplies will be out of stock, or that they will be poorly treated (3).

Addressing the different causes of maternal mortality requires well-functioning health system that encompasses all levels - from the community to referral facilities and provides accessible, good quality care. Adequate supplies, skilled personnel, and an effective system for referral and transport are particularly important for managing obstetric emergencies, which can arise suddenly and without warning. Efforts addressing underlying factors are also important to improve maternal health in the long term. Interventions are required with families \& communities, in society as a whole, in health system, and at the national legislation \& policy levels. Some of the recommendations are ensuring skilled birth attendance at delivery; prevention of unwanted pregnancy and unsafe abortion; recognize that every pregnancy faces risk and improve access to good quality maternal health services; addressing barriers to access and measure progress made.

\section{Reference}

1. WHO, UNFPA, UNICEF, World Bank. Reduction of maternal mortality: A joint WHO/UNFPA/UNICEF/World Bank statement. Geneva: WHO; 1999.

2. Starrs A. The safe motherhood action agenda: Priorities for the next decade-Report on the Safe Motherhood Technical Consultation, 18-23 October 1997, Colombo, Sri Lanka.

3. Thaddeus S, Maine D. Too far to walk: Maternal mortality in context: Findings from a multidisciplinary literature review. Columbia University School of Public Health: Prevention of Maternal Mortality Program, Center for Population and Family Health, 1990.

4. World Health Organization. Maternal mortality in 2005, estimates developed by WHO, UNICEF, UNFPA and The World Bank [Cited 2010 May]: Available from: URL:http://www.who.int/reproductive-health/ publications/maternal_mortality_2005/mme_2005.p $\underline{\mathrm{df}}$

5. Central Statistical Authority, ORC Macro. Ethiopia Demographic and Health Survey: 2000. Addis Ababa, Ethiopia: 2001.

6. Central Statistical Authority, ORC Macro. Ethiopian Demographic and Health Survey: 2005. Addis Ababa, Ethiopia: 2005.

7. Amanuel G. Maternal complications - in a zonal hospital. Ethiop Med J. 2007; 45(1):47-54.

8. Kwast BE, Kidane-Mariam W, Saed EM, Fowkes FG. Epidemiology of maternal mortality in Addis Ababa: a community-based study. Ethiop Med J 1985; 23:7-16.

9. Kwast BE. Unsafe motherhood: A monumental challenge-A study of maternal mortality in Addis Ababa. Netherland: Lithoprint Ltd (TU), 1988.

10. Tadesse E, Yoseph S, Gossa A. Survey of abortion in 5 public hospitals in the city of Addis Ababa. Unpublished: 1994.

11. Seyom Y, Getahun K. A six year review of maternal mortality in a teaching hospital in Addis Ababa. Ethiop.Med. J. 1988; 26:115-9.

12. Berhan Y, Abdella A. Emergency obstetrics performance with emphasis on operative delivery outcome: Does it reflect the quality of care. Ethiop J Health Dev 2004; 18(2):96-106.

13. Abdella A. Maternal mortality in two teaching hospitals in Addis Ababa, Ethiopia, 2007-2008. Unpublished.

14. Berhanu H, Almaguer M. Alfonzo Guerra JP, Aree S. Clinical profile of acute renal failure in an Addis Ababa referral hospital. Ethiop Med J 1984; 22(4):179-64.

15. Getachew A. Outcome of pregnancies complicated with preeclampsia-eclampsia at St. Paul's HospitalCase control study.

16. Misganaw A, Lakew Z. Eclampsia: A 5 years retrospective review of 216 cases managed in two teaching hospitals in Addis Ababa. Ethiop. Med. J. 2006; 44(1):27-32.

17. Sisay M, Korsak V, Mekonnen B, Perfilieva G. Criminal abortion: Six year review in St. Paul's Hospital. XXIII Annual Medical Conference.

18. Herpassa Garomossa, A.D. Dwivedi. Maternal mortality in Ambo Hospital: a five year retrospective review. Ethiop. J. Reproductive Health 2008; 2(1):213.

19. Mekbib T, Ketsela K. Preeclampsia/eclampsia at Yekatit 12 Hospital, Addis Ababa, Ethiopia. East Afr. Med. J. 1991; 68:893.

20. Tigray Regional Health Bureau. Tigray Regional Health Bureau. Maternal and child mortality in Tigray Region, 2003: Report of a community based survey. Tigray Regional Health Bureau, 2004.

21. Ali Y. Analysis of maternal deaths in Jimma Hospital, Southwestern Ethiopia. Ethiop. Med. J. 1994; 32(2):125-29.

22. Federal Democratic Republic of Ethiopia. The criminal code of conduct of the Federal Democratic

Ethiop. J. Health Dev. 2010;24 Special Issue 1 
Republic of Ethiopia. Proclamation No. 414/2004 2005.

23. Zewodu W. Acute renal failure in Addis Ababa, Ethiopia: A prospective study of 136 patients. Ethiop. Med. J. 1994; 32(2):79-87.

24. Frost O. Maternal and perinatal death in an Addis Ababa hospital. Ethiop. Med J. 1984; 35:114-8.

25. Ministry of Health, Federal Democratic Republic of Ethiopia. Technical and Procedural Guideline for Safe Abortion Services in Ethiopia. Addis Ababa, Ethiopia: 2006.

26. Tsega E. Current views in liver disease in Ethiopia. Ethiop. Med. J. 1977; 15:75-82.

27. Tsega E. Viral hepatitis in pregnancy in Ethiopia. East Afr. Med. J. 1976; 53:270-7.

28. Tsega E, K. Krawczynski, BG. Hansson, E. Nordenfelt. Hepatitis E Virus infection in pregnancy in Ethiopia. Ethiop. Med. J. 1993; 31(3):173-81.

29. Alemu A., Abrens C. Baseline survey of female reproductive health: Western Zone, Tigray Region. 2001:(Unpublished).

30. Ferguson A. Baseline survey of female reproductive health: Southern Zone, Tigray Region. 2000:(unpublished).

31. Tesfaye Shiferaw, Fasil Tessema. Maternal mortality in rural communities of Illubabor, Southwestern Ethiopia as estimated by the sisterhood method. Ethiop. Med. J. 1993; 31(4):239-51.

32. World Health Organization. Maternal mortality ratios and rates: A tabulation of available information $3^{\text {rd }}$. Division of Family Health, Geneva: WHO; 1991.

33. Berhane Y. Women's health and reproductive outcome in rural Ethiopia. Department of Community Health and Epidemiology Department of Public Health and Clinical Medicine, Addis Ababa University and Umea University: Umea University, SE-901 85 Umea, Sweden, 2000. p. 3334.
34. Mehari S. Grand multiparity at Jimma Hospital. Ethiop J Health Sciences 1999; 9(1):41-6.

35. Mekbeb TA, Gebre Hiwot Y. Misganaw A. Survey of unsafe abortion in selected health facilities in Ethiopia. Ethiop. J. Repro. Health 2007; 1(1):28-43.

36. Tesfaye M, Tinbit GT. A six month prospective study on different aspects of abortion. Ethiop. Med. J. 1993; 31(3):165-72.

37. Jackson AP. Eclampsia in Addis Ababa: Pattern and treatment. Ethiop. Med. J. 1970; 8:123-5.

38. Gessesew A, Mesfin M. Ruptured uterus: Eight year retrospective analysis of causes and management outcome in Adigrat Hospital, Tigray Region, Ethiopia. Ethiop. J. Health Dev. 2002; 16(3):241-45.

39. Abate M. Eclampsia: A retrospective review in tow teaching hospitals in Addis Ababa. 1999:Unpublished.

40. Gaym A. Obstructed labor at a district hospital. Ethiop. Med. J 2002; 40(1):11-8.

41. Chamisso B. Rupture of the pregnant uterus in Shshemene General Hospital. Ethiop. Med. J. 1995; 33(44):251-57.

42. Eyob B, Gaym A. Risk factors for mortality among eclamptics admitted to the surgical intensive care unit at Tikur Anbessa Hospital, Addis Ababa, Ethiopia. Ethiop. J. Repro. Health 2007; 1(1):4-16.

43. Ahmed A. Retrospective study on abortion admissions in Jimma Hospital, Southwest Ethiopia. Ethiop. J. Health Dev. 1996; 10(1):167-70.

44. Asheber G. A review of maternal mortality at Jimma Hospital, Southwestern Ethiopia. Ethiop J Health Dev 2000; 14(2):215-23.

45. Teklu S, Gaym A. Prevalence and clinical correlates of the hypertensive disorders of pregnancy at Tikur Anbessa Hospital. Ethiop. Med. J. 2006; 44(1):17-26. 